\title{
KNOWLEDGE OF HEALTH EFFECTS OF DIETARY FIBRE AMONG NURSES AND THE GENERAL POPULATION IN CROATIA: COMPARATIVE ANALYSIS
}

\author{
Marija Ljubicic, ${ }^{1}$ Marijana Matek Saric, ${ }^{2}$ Katja Curin, ${ }^{3}$ Raquel P. F. Guine, ${ }^{4}$
}

\begin{abstract}
:
Introduction: Nurses play diverse roles in educating patients about a healthy diet and in promoting healthy eating habits, through their conversations with patients and families and through their work providing enteral and parenteral feeding to critically ill patients. Given the position of nurses as key providers of dietary guidance, and given the health benefits of dietary fibre, we wanted to assess the level of fibre-related knowledge among nurses in Croatia.

Objectives: The primary objective of this cross-sectional s tudy was to compare knowledge between nurses and the general population from Croatia about the health importance of consuming dietary fibre.

Methods: Knowledge from 369 nurses and 727 residents from the general population about fibre was assessed using a validated survey developed by the CI\&DETS Polytechnic Institute, Viseu, Portugal. Differences between nurses and the general population were assessed for significance using the Mann-Whitney $U$ test and the Kruskal-Wallis test, while associations between study variables and knowledge were explored using Spearman rank correlation coefficients.

Results: The level of knowledge among nurses and the general population in Croatia about dietary fibre varies from «undecided» to «partial knowledge». No association was observed between nurse education level and either general knowledge about fibre or knowledge about the health benefits of fibre intake. No significant differences were observed between nurses and the general population in general knowledge $(p=0.894)$ or in health-related knowledge $(p=0.578)$.

Conclusion: The results suggest the need for updating and expanding nursing curricula. The similar level of knowledge between nurses and the general population about dietary fibre indicates the need to strengthen nurse education and training in the areas of diet and diet therapy in Croatia. Given the role of nurses as diet educators and advisors, this may help improve the quality of health care.
\end{abstract}

UDC Classification: 616-01; DOI: http://dx.doi.org/10.12955/cbup.v5.1053

Keywords: knowledge, dietary fiber, nurses, general population.

\section{Introduction}

Public health campaigns and interventions designed to promote healthy behaviors such as diet often assume that if people know what is good for them, they are more likely to act in their own best health interest (Worsley, 2002). However, although knowledge about diet is indeed a predictor of healthy food consumption, it is not the only predictor (Worsley, 2002). Consumption of healthy food in general, and intake of dietary fibre in particular, also depends on knowledge, habits, motivation, personal characteristics and hedonistic orientation. This has important implications for health professionals, whose mission is to promote positive health behaviours through effective education, counselling and awareness-building.

Numerous studies have demonstrated the health benefits of dietary fibre, including its ability to prevent and treat various diseases (Fuller et al., 2016; Galisteo et al., 2008; Hajishafiee et al., Esmaillzadeh, 2016; Ismaiel et al., 2016; Kunzmann et al., 2015). Dietary fibre can be defined as edible parts of plants that are not digestible in the small intestine and so are not absorbed there, but that are partially or totally degraded in the large intestine by intestinal microflora (Guine, Martinho, Barroca, \& Viseu, 2014). Main sources of dietary fibre are vegetables, fruit, grains, legumes and seeds. Daily intake of $25 \mathrm{~g}$ of dietary fibre is important for optimal functioning of the intestines, and daily intake above this amount can reduce the risk of many chronic diseases (Slavin, 2013; Yang, et al., 2015).

Knowledge about the health effects of fibre, daily intake requirements and methods to monitor fibre intake is crucial for nurses to provide effective care to patients with high comorbidity burdens. Indeed, nurses play diverse roles in educating patients about healthy diets and in promoting healthy eating habits, through their conversations with patients and families and through their work providing enteral and parenteral feeding to critically ill patients (Green \& Jackson, 2002; Lazarou \& Kouta, 2010). Nurses are able to identify health gaps in patient knowledge and behaviour or lifestyle, and they can

\footnotetext{
${ }^{1}$ University of Zadar, General Hospital Zadar, Croatia, marija.ljubicic.zadar@gmail.com

${ }^{2}$ University of Zadar, Department of Health studies, Zadar, Croatia, marsaric@unizd.hr

${ }^{3}$ Institute for Public Health of Split - Dalmatia County, Split, Croatia, dr.katja.curin@ gmail.com

${ }^{4}$ CI\&DETS, Polytechnic Institute of Viseu, Portugal, raquelguine@esav.ipv.pt
} 
react accordingly. In fact, they are well-placed to design research to examine these health gaps and develop solutions for them (Brotheton et al., 2013). Florence Nightingale emphasized the role of diet in patient recovery, and the important role of nurses in supporting proper diet (Yalcin et al., 2013). Nurses are frequently called upon to act as dietary consultants, offering patients information about diet and about healthy food selection (Henning, 2009), as well as monitoring patients' food intake and eating habits. These duties come in addition to the routine duties of care such as administering treatments, monitoring vital signs and conducting other interventions (Yalcin et al., 2013). Education provides the foundation for a nurse to plan, provide and evaluate dietary care (Green \& Jackson, 2002).

We are unaware of published studies assessing knowledge about dietary fibre among nurses, and it appears that in Croatia, no study has been published on nurses' knowledge of diet in general or of fibre in particular. Research into such knowledge levels may generate key insights for improving systematic training and education of nurses. Given the position of nurses as key providers of dietary guidance, and given the health benefits of dietary fibre, we wanted to assess the level of fibre-related knowledge among nurses in Croatia and compare it with the level of knowledge in the general population. We examined both general knowledge about fibre and specific knowledge about the demonstrated effects of fibre on human health. In light of the research literature from other countries, we hypothesized that nurses in Croatia would show higher levels of knowledge than the general population.

The results of our study may help guide the strengthening of dietary training at all levels of nursing education and training, which in turn may improve the ability of nurses to prevent and treat disease through diet therapy.

\section{Methods}

\section{Design and Samples}

In this cross sectional study, the level of knowledge about dietary fibre was assessed using a validated survey developed by the CI\&DETS Research Centre at the Polytechnic Institute Viseu in Portugal (7). The survey contains questions about demographic characteristics as well as about knowledge of sources of dietary fibre, recommended daily intake and effects of fibre intake on particular diseases. They were asked about health knowledge i.e. the influence of fibre on the risk of cardiovascular disease, hypercholesterolemia, diabetes, obesity, constipation, carcinoma of the colon or breast, vision problems and vitamin and mineral deficiency.

This study was approved by the Human Research Ethics Committee of the General Hospital, Home Health and Nursing school. Aims of the study were explained to all participants and they were assured that participation was voluntary and anonymous. Upon providing consent, respondents filled out the questionnaires on their own and handed them in by placing them in a marked box.

Participants

A total of 1,096 respondents, comprising 369 nurses and 727 residents from the general population, were surveyed on the coast of Croatia. Nurses were from three health centres and one nursing school, all located in Zadar County, Dalmatia. Respondents were 69.7\% female (764) and 30.9\% male (332).

Among the respondents in the general population, 57.3\% (417) were female and $42.6 \%$ (310) were male; the corresponding proportions among nurse respondents were $94.0 \%$ (347) and $5.9 \%$ (22). The preponderance of female nurses contributed to the high proportion of females in the overall sample. An urban environment was the living situation for most respondents from the general population (551, $77 \%)$ as well as for most nurses $(268,74.4 \%)$, while $165(23 \%)$ respondents from the general population and $92(25.6 \%)$ nurses lived in a rural environment.

Average age of respondents was 38.9 years (SD 12.3; 95\% CI 38.2-39.6), ranging from 18 to 77 years. Most respondents from the general population and most nurses had a secondary school education.

Measures

Respondents indicated their level of agreement with statements about fibre using a Likert scale from 1 to 5: 1-completely disagree; 2-disagree; 3-neither agree or disagree; 4-agree; 5-completely agree. These levels of agreement were then transformed into levels of knowledge, such that responses of 5 indicated complete knowledge; 4, partial knowledge; 3, indecision; 2, partial lack of knowledge; and 1 , complete lack of knowledge. Levels of agreement with questions formulated in the negative, such that 5 indicated complete agreement with an incorrect statement, were recoded before calculating level 
of knowledge. As a result, levels of knowledge always indicated correct knowledge. Levels of knowledge were converted to percentages by multiplying central values by $20(1=20 \%)$. Test reliability was good (Cronbach's $\alpha=0.775$ ).

Data analysis

SPSS 22.0 (IBM, Armonk, NY, USA) was used to generate descriptive statistics about the results (frequency distributions, central tendency measures, dispersion measures) and to perform inferential statistical analysis to assess influences of sex, age, living environment and education level on knowledge about dietary fibre. Test reliability was assessed using Cronbach's alpha, and results were tested for normal distribution using the Kolmogorov-Smirnov test. This test indicated a skewed distribution, so inter-group differences were assessed using the non-parametric Mann-Whitney $U$ and Kruskal-Wallis tests. Associations between study variables and knowledge about dietary fibre were explored using a Spearman rank correlation analysis. For all statistical analyses, the threshold of significance was defined as $p<0.05$.

\section{Results}

The survey asked respondents about their knowledge of recommended daily intake of dietary fibre and the presence and abundance of fibre in various foods and ability of dietary fibre intake to help prevent certain diseases. The median central value for level of knowledge of the various items ranged from 3 to 4 (interquartile range $\left(\mathrm{I}_{\mathrm{q}}\right)$ was 1 to 2 ) for the general population and for nurses. Relatively strong variability $\left(\mathrm{V}_{\mathrm{q}} 0.33\right)$ was observed among both group on items asking about the presence of dietary fibre in foods of plant or animal origins. Both groups showed low variability in knowledge on all items related to the health effects of dietary fibre, with $\mathrm{V}_{\mathrm{q}}$ ranging from 0.00 to 0.20 . Average levels of general knowledge about fiber in percentages ranged from $58.9 \%$ to $83 \%$ for the general population, and from $61.4 \%$ to $82.1 \%$ for nurses, and $57.6 \%$ to $82.0 \%$ for nurses and from $58.9 \%$ to $79.3 \%$ for the general population for knowledge about the health importance of dietary fibre.

Possible associations were explored between general knowledge about dietary fibre and several sociodemographic factors: age, sex, education level and living environment. Among nurses, those aged 36 to 45 showed significantly higher general knowledge than other age groups $(p=0.013)$. No other significant associations were observed. None of the sociodemographic factors appeared to significantly influence levels of knowledge about the dietary effects of fibre intake.

Among respondents from the general population, general knowledge was significantly higher among those living in urban environments compared to those in rural environments $(p=0.002)$, among those with higher education levels compared to those with less education $(p=0.042)$ and among women compared to men $(p=0.022)$. No other significant associations were observed. The only significant association identified between level of knowledge about health effects of fibre and sociodemographic factors was sex, with women showing significantly higher health-related knowledge than men $(p=$ $0.004)$.

Respondents from the general population with higher education levels showed significantly higher levels of knowledge about the presence of dietary fibre in whole grain foods $(1.6 \%, \mathrm{r}=0.13, p=$ $0.001)$ and unpeeled fruit $(0.5 \%, \mathrm{r}=0.08, p=0.045)$, and about the influence of fibre intake on the risk of bowel cancer $(0.6 \%, \mathrm{r}=0.08, p=0.032)$. No significant association was observed between education level and knowledge level on the other items.

Among nurses, those with higher education levels showed significantly higher knowledge levels about the presence of dietary fibre in whole grain foods $(1.2 \%, \mathrm{r}=0.11, p=0.035)$ and about the influence of fibre intake on the risk of certain diseases $(1.9 \%, r=0.14, p=0.007)$, constipation $(1.6 \%, r=0.13$, $p=0.016)$ and specifically the risk of bowel cancer $(2.1 \%, \mathrm{r}=0.15, p=0.005)$ and breast cancer $(1.2 \%, \mathrm{r}=0.11, p=0.039)$.

In both groups, significant associations were identified between general knowledge about dietary fibre and knowledge about its health benefits (Table 1). Education level was associated with level of general knowledge among respondents from the general population, but it was not associated with general or health-related knowledge among nurses. 
Table 1: Association between education level and level of general or health-specific knowledge about dietary fibre $(\mathrm{N}=$ $1,096)$

\begin{tabular}{|c|c|c|c|c|c|}
\hline & \multicolumn{2}{|c|}{ General population } & \multicolumn{2}{|c|}{ Nurses } \\
\hline & & \multirow{2}{*}{\begin{tabular}{r}
\multicolumn{1}{c}{$\begin{array}{c}\text { Health } \\
\text { knowledge }\end{array}$} \\
$0.192^{* *}$
\end{tabular}} & \multirow{2}{*}{$\begin{array}{c}\text { Education level } \\
0.078^{*}\end{array}$} & \multirow{2}{*}{$\begin{array}{r}\text { Health knowledge } \\
0.293^{* *}\end{array}$} & \multirow{2}{*}{$\begin{array}{r}\text { Education level } \\
0.101\end{array}$} \\
\hline General & $\mathrm{r}_{\mathrm{s}}$ & & & & \\
\hline knowledge & $\mathrm{p}$ & 0.000 & 0.042 & 0.000 & 0.064 \\
\hline \multirow{2}{*}{$\begin{array}{l}\text { Health } \\
\text { knowledge }\end{array}$} & $\mathrm{r}_{\mathrm{s}}$ & & 0.019 & & 0.097 \\
\hline & $\mathrm{p}$ & & 0.622 & & 0.080 \\
\hline
\end{tabular}

Source: Author

The general population showed higher knowledge about the absence of dietary fibre from foods of animal origin, and about the ability of dietary fibre intake to prevent and treat certain diseases in general. Nurses showed higher knowledge about the ability of dietary fibre intake to prevent and treat obesity, constipation and breast cancer in particular (Tables 2). No other differences in knowledge between the two groups were observed on other items.

\begin{tabular}{|c|c|c|c|}
\hline Survey item & Sample & Mean Rank ${ }^{\mathrm{a}}$ & $\mathbf{p}^{\mathrm{b}}$ \\
\hline \multirow{2}{*}{ Dietary fibre can prevent and treat diseases } & general population & 563.26 & \multirow{2}{*}{0.003} \\
\hline & nurses & 510.37 & \\
\hline \multirow{2}{*}{ Dietary fibre can prevent and treat obesity } & general population & 527.86 & \multirow{2}{*}{0.018} \\
\hline & nurses & 571.57 & \\
\hline \multirow{2}{*}{ Dietary fibre can prevent and treat breast cancer } & general population & 519.30 & \multirow{2}{*}{0.004} \\
\hline & nurses & 573.10 & \\
\hline \multicolumn{4}{|l|}{$\begin{array}{l}{ }^{\mathrm{a}} \text { Mann-Whitney U test } \\
{ }^{b} \mathrm{p}<0.05\end{array}$} \\
\hline Source: Author & & & \\
\hline
\end{tabular}

Aggregating responses on all items about general knowledge of dietary fibre or on all items about health-related effects of fibre intake indicated higher levels of both types of knowledge among nurses than among respondents from the general population though the differences were not significant (Table 3).

Table 3 : Differences in knowledge about dietary fibre between nurses and the general population $(\mathrm{N}=1,096)$

\begin{tabular}{|l|l|r|r|}
\hline \multirow{2}{*}{ Survey item } & Sample & Mean Rank & \multirow{2}{*}{$\mathbf{p}^{\mathrm{b}}$} \\
\hline \multirow{2}{*}{ General knowledge } & general population & 517.63 & \multirow{2}{*}{0.894} \\
\cline { 2 - 3 } & nurses & 520.27 & \\
\hline \multirow{2}{*}{ Health knowledge } & general population & 510.96 & \multirow{2}{*}{0.578} \\
\cline { 2 - 3 } & nurses & 521.98 & \\
\hline
\end{tabular}

${ }^{a}$ Mann-Whitney U test

${ }^{b} \mathrm{p}<0.05$

Source: Author 


\section{Discussion}

Motivated by the large number of studies demonstrating the positive health effects of dietary fibre in the diet therapy of many diseases, we set out to assess the levels of knowledge about fibre in general and its health benefits in particular among nurses and the general population in Croatia. Our results indicate that although nurses often recommend consuming fibre to treat conditions such as constipation, they have substantial gaps in knowledge about the effects of dietary fibre intake on health. Indeed, we found that nurses had nearly the same level of knowledge about dietary fibre as the general population. At the same time, nurses showed higher levels of specific knowledge about the influence of fibre intake on constipation, obesity and carcinoma of the colon or breast. Levels of knowledge in both groups varied substantially.

In our research and other studies, a high proportion of nurses have limited knowledge about diet therapy and about sources of soluble fibre and other foods that can prevent or treat certain diseases (Park et al., 2011). However, our finding that nurses showed higher levels of specific knowledge about the influence of fibre intake on constipation, obesity and carcinoma of the colon or breast likely reflects their formal education and previous experience. Our finding of similar knowledge points to inadequacies in nurse knowledge, which can negatively affect patient care. Studies in different countries point to deficits in dietary knowledge among not only nurses but other health professionals as well in South Korea and Australia (Kowanko et al., 1999; Park et al., 2011; Schaller \& James, 2005); Denmark, Sweden and Norway (Mowe et al., 2008). In addition, the inadequate number of dieticians and nutritionists in the health system means that nurses often shoulder the responsibility of dietary advisor (Brotheton et al., 2013). However, nurses lack the necessary dietary education to offer optimal guidance and advice to patients (Henning, 2009; Kowanko et al., 1999; Yalcin et al., 2013). Nursing curricula include diet as only a small part of the wider spectrum of nursing areas, which is inadequate for satisfying patient needs in practice (Green \& Jackson, 2002; Kowanko et al., 1999). This lack of dietary knowledge makes it difficult for nurses and other medical staff to identify malnourished patients and correctly calculate a patient's energy requirements. These deficiencies may contribute to the risk of complications and prolong patient stay in hospital (Mowe et al., 2008).

Studies demonstrate that nurses are best positioned to provide patients with all necessary information about diet (O’Mahony et al., 2011; Yalcin et al., 2013). Analysis of nursing experiences indicates that patients frequently ask them to provide dietary information. Studies show that nurses are in constant contact with patients and continuously communicate with them (O'Mahony et al., 2011) and often receiving dietary questions from patients, but nurses are often busy with other tasks, such as documentation and medication (Eide et al., 2015). Nurses accompany patients throughout their health care to prevent and treat disease, so their knowledge about diet and dietary fibre is key to successful diet therapy, which is an essential part of a patient's non-pharmacological treatment (Rodrigues-Fisher, Bourguignon, \& Good, 1993). Nurses require a certain level of dietary knowledge to advise patients about choice of foods, meal sizes and meal schedules, all of which play an important role in diet therapy. Numerous studies underscore how important it is for a nurse to understand diet and the influence of specific ingredients in food on health (Brotheton et al., 2013; Kowanko et al., 1999; Park et al., 2011; Yalcin et al., 2013). as well as to be able to assess nutritional patterns according to Marjory Gordon (Gordon, 2011). However, studies show that nurses often lack this necessary knowledge and rarely document assessments of diet status, energy intake and body mass indexs (Kim \& Choue, 2009; Persenius et al., 2008). Instead, nurses tend to note only the appearance of difficulties such as nausea, vomiting, reduced mobility or eating ability; assessment of diet status is usually limited to addressing patient concerns about specific types of foods (Persenius et al., 2008). This incomplete dietary assessment can lead to incorrect estimation of dietary needs, which can negatively affect patient recovery.

Our findings are consistent with literature showing that, even though nurses and physicians emphasise the importance of diet in medical care, and even though patients expect to receive relevant dietary information from their healthcare providers, the reality is that healthcare workers often feel poorly equipped to offer dietary guidance (Adams, Kohlmeier, Powell, \& Zeisel, 2010). One study even found that physicians knew less than their patients about some dietary topics (Lazarus, 1997).

Several studies have shown that nurses as well as other health professionals often have insufficient dietary knowledge (Adams et al., 2010; Lane et al., 2014; Lazarus, 1997; Mowe et al., 2008; Warber, 
et al., 1997; Yalcin et al., 2013). Park et al found that although most nurses are aware of the need for diet therapy, they have limited knowledge about low-cholesterol diets, sources of soluble fibre and specific foods that can help prevent disease (Park et al., 2011). Those authors concluded that nurses, had weak knowledge about clinical aspects of diet. This suggests that medical staff pay insufficient attention to diet, despite the fact that diet therapy is an important part of patient care.

Education appears to be more important than experience in providing good dietary care to patients: studies have shown that experience is not a predictor of dietary knowledge, and that less experienced nurses can showed greater knowledge than their more senior colleagues (Crogan \& Evans, 2001). However, in our study, we observe a significant association between education level and knowledge about dietary fibre. Numerous studies concluding that higher education level among nurses is associated with greater dietary knowledge (Yalcin et al., 2013), and that they need education in the areas of diet therapy and many other diseases (Bjerrum et al., 2012; Crogan et al., 2001; Endevelt et al., 2009; Warber et al., 1997). We suspect that this discrepancy reflects the relatively high proportion of nurses in our sample with secondary education, as well as the relatively low levels of knowledge among university-educated nurses in our sample. On balance, the literature suggests a positive effect of education on knowledge about dietary fibre. Therefore, our results and those of others highlight an urgent need to update and expand content related to diet and diet therapy in the nursing curriculum (Boaz et al., 2013; Mowe et al., 2008; Park et al., 2011; Yalcin et al., 2013).

Studies examining questions of nurse knowledge and experience should be careful to distinguish between the two, which the literature often associates or even treats as equivalent. The two concepts are not necessarily inter-related: studies show that experience in the absence of specific education may not increase the level of knowledge about diet (Crogan et al., 2001; Yalcin et al., 2013) or dietary fibre.

Despite the strengths of the present study, including its relatively large sample, good test reliability, and analysis of different types of knowledge about dietary fibre, the work has some limitations. The survey did not collect data that would allow us to determine the percentage of respondents from the general population who might be more likely to know more about dietary fibre than others, such as scientists, physicians, and nutritionists.

Furthermore, the strong influence that nurses exert on patients' attitudes toward diet and healthy behaviours means that adequate dietary knowledge is crucial. The results of the present study, together with numerous studies from other countries, suggest the need for curriculum reform at all levels of nursing to strengthen knowledge of diet, diet therapy and health benefits of dietary fibre.

\section{Conclusion}

While the nurses in our sample showed a certain level of knowledge about the influence of dietary fibre intake on prevention and treatment of certain diseases, on the whole the nurses showed levels of knowledge about dietary fibre similar to those in the general population. This is troubling because nurses, given their role as diet educators and advisors, should know more about the subject than their patients. These results suggest the need for updating and expanding nursing curricula in the areas of diet and diet therapy.

\section{References}

Adams, K., Kohlmeier, M., Powell, M., \& Zeisel, S. (2010). Nutrition in medicine: nutrition education for medical students and residents. Nutr Clin Pract 25, 471-480.

Bjerrum, M., Tewes, M., \& Pedersen, P. (2012). Nurses' self-reported knowledge about and attitude to nutrition - before and after a training programme. Scand J Caring Sci, 26, 81-89.

Boaz, M., Rychani, L., Barami, K., Hour, Z., Yosef, R., Siag, A. et al. (2013). Nurses and nutrition: a survey of knowledge and attitudes regarding nutrition assessment and care of hospitalized elderly patients. J Contin Educ Nurs, 44, 357-364.

Brotheton, C., Taylor, A., \& Anderson, J. (2013). Nurse-led investigation of the relationship between dietary fiber and Crohn's disease symptoms. Inflamm Bowel Dis, 19, S7-S8.

Crogan, N., \& Evans, B. (2001). Nutrition assessment: experience is not a predictor of knowledge. J Contin Educ Nurs, 32, 219-222.

Crogan, N., Shultz, J., \& Massey, L. (2001). Nutrition knowledge of nurses in long-term care facilities. J Contin Educ Nurs, $32,171-176$.

Eide, H. D., Halvorsen, K., \& Almendingen, K. (2015). Barriers to nutritional care for the undernourished hospitalised elderly: perspectives of nurses. J Clin Nurs, 24(5-6), 696-706. 
Endevelt, R., Werner, P., Goldman, D., \& Karpati, T. (2009). Nurses' knowledge and attitudes regarding nutrition in the elderly. J Nutr Health Aging, 13, 485-489.

Fuller, S., Beck, E., Salman, H., \& Tapsell, L. (2016). New horizons for the study of dietary fiber and health: a review. Plant Food Hum Nutr 71, 1-12.

Galisteo, M., Duarte, J., \& Zarzuelo, A. (2008). Effects of dietary fibers on disturbances clustered in the metabolic syndrome. J Nutr Biochem, 19, 71-84.

Gordon, M. (2011). Manual of nursing diagnosis. Sudbury-Massachusetts: Jones \& Bartlett Publishers.

Green, S., \& Jackson, P. (2002). Nutrition and nurse education. Nur Times, 98, 59.

Guine, R., Martinho, C., Barroca, M., \& Viseu, C. (2014). Knowledge and attitudes regarding dietaryfibres: a consumer survey in portuguese population. JBARI 1, 1-12.

Hajishafiee, M., Saneei, P., Benisi-Kohansal, S., \& Esmaillzadeh, A. (2016). Cereal fibre intake and risk of mortality from all causes, CVD, cancer and inflammatory diseases: a systematic review and meta-analysis of prospective cohort studies. $\mathrm{Br} J$ Nutr, 116, 343-352.

Henning, M. (2009). Nursing's role in nutrition. Comput Inform Nurs, 27, 301-306.

Ismaiel, M., Yang, H., \& Min, C. (2016). Dietary fiber role in type 2 diabetes prevention. Brit Food J 118, 961-975.

Kim, H., \& Choue, R. (2009). Nurses' positive attitudes to nutritional management but limited knowledge of nutritional assessment in Korea. Int Nurs Rev 56, 333-339.

Kowanko, I., Simon, S., \& Wood, J. (1999). Nutritional care of the patient: nurses' knowledge and attitudes in an acute care setting. J Clin Nurs, 8, 217-224.

Kunzmann, A., Coleman, H., Huang, W., Kitahara, C., Cantwell, M., \& Berndt, S. (2015). Dietary fiber intake and risk of colorectal cancer and incident and recurrent adenoma in the prostate, lung, colorectal, and ovarian cancer screening trial. $A m$ J Clin Nutr, 102, 881-890.

Lane, C., Wedlake, L., Dougherty, L., \& Shaw, C. (2014). Attitudes towards and knowledge of nutrition support amongst health care professionals on London intensive care units. J Hum Nutr Diet, 27, 339-351.

Lazarou, C., \& Kouta, C. (2010). The role of nurses in the prevention and management of obesity. Br J Nurs 19, 641-647.

Lazarus, K. (1997). Nutrition practices of family physicians after education by a physician nutrition specialist. Am J Clin Nutr 65, 2007S-2009S.

Mowe, M., Bosaeus, I., Rasmussen, H., Kondrupd, J., Unossone, M., Rothenbergb, E. et al. (2008). Insufficient nutritional knowledge among health care workers? Clin Nutr, 27, 196-202.

O'Mahony, S., Hutchinson, J., McConnell, A., Mathieson, H., \& McCarthy, H. (2011). A pilot study of the effect of a nutrition education programme on the nutrition knowledge and practice of nurses. J Hum Nutr Diet 24, 300.

Park, K., Cho, W., Song, K., Lee, Y., Sung, I., \& Choi-Kwon, S. (2011). Assessment of nurses' nutritional knowledge regarding therapeutic diet regimens. Nurse Educ Today, 31, 192-197.

Persenius, M., Hall-Lord, M., Bååth, C., \& Larsson, B. (2008). Assessment and documentation of patients' nutritional status: perceptions of registered nurses and their chief nurses. J Clin Nurs, 17, 2125-2136.

Rodrigues-Fisher, L., Bourguignon, C., \& Good, B. (1993). Dietary fiber nursing intervention prevention of constipation in older adults. Clin Nurs Res, 2, 464-477.

Schaller, C., \& James, E. (2005). The nutritional knowledge of Australian nurses. Nurse Educ Today, 25, 405-412.

Slavin, J. (2013). Fiber and prebiotics: mechanisms and health benefits. Nutrients 5, 1417-1435.

Warber, J., Simone, K., \& Waber, J. (1997). Assessment of nutrition knowledge of nurse practitioners in New England. $J$ Acad Nutr Diet, 97, A112.

Worsley, A. (2002). Nutrition knowledge and food consumption: can nutrition knowledge change food behaviour? Asia Pac J Clin Nutr, 11, 579-585.

Yalcin, N., Cihan, A., Gundogdu, H., \& Ocakci, A. (2013). Nutrition knowledge level of nurses. Health Science Journal, 7, 99-108.

Yang, Y., Zhao, L., Wu, Q., Ma, X., \& Xiang, Y. (2015). Association between dietary fiber and lower risk of all-cause mortality: a meta-analysis of cohort studies. Am J Epidemiol 181, 83-91. 